\title{
Hepatoprotective Effects of Chinese Medicine Herbs Decoction on Liver Cirrhosis in Rats
}

\author{
Nor Aziyah Mat-Rahim, ${ }^{1,2}$ Tong-Hye Lim, ${ }^{3}$ \\ Nur-Asyura Nor-Amdan, ${ }^{1}$ and Sazaly AbuBakar ${ }^{1}$ \\ ${ }^{1}$ Tropical Infectious Diseases Research and Education Center (TIDREC), Department of Medical Microbiology, \\ Faculty of Medicine, University of Malaya, 50603 Kuala Lumpur, Malaysia \\ ${ }^{2}$ Virology Unit, Institute for Medical Research, Jalan Pahang, 50588 Kuala Lumpur, Malaysia \\ ${ }^{3}$ Herbitec (M) Sdn Bhd, 11-2 Jalan Sri Hartamas 7, Taman Sri Hartamas, 50480 Kuala Lumpur, Malaysia \\ Correspondence should be addressed to Sazaly AbuBakar; sazaly@um.edu.my
}

Received 20 July 2016; Accepted 16 January 2017; Published 9 February 2017

Academic Editor: Luciana Dini

Copyright (C) 2017 Nor Aziyah Mat-Rahim et al. This is an open access article distributed under the Creative Commons Attribution License, which permits unrestricted use, distribution, and reproduction in any medium, provided the original work is properly cited.

\begin{abstract}
Hepatoprotective and curative activities of aqueous extract of decoction containing 10 Chinese medicinal herbs (HPE-XA-08) were evaluated in Sprague-Dawley albino rats with liver damage induced by thioacetamide (TAA). These activities were assessed by investigating the liver enzymes level and also histopathology investigation. Increases in alkaline phosphatase (ALP) and gammaglutamyl transferase (GGT) levels were observed in rats with cirrhotic liver. No significant alterations of the liver enzymes were observed following treatment with HPE-XA-08. Histopathology examination of rats treated with HPE-XA-08 at $250 \mathrm{mg} / \mathrm{kg}$ body weight, however, exhibited moderate liver protective effects. Reduced extracellular matrix (ECM) proteins within the hepatocytes were noted in comparison to the cirrhotic liver. The curative effects of HPE-XA-08 were observed with marked decrease in the level of ALP (more than 3x) and level of GGT (more than 2x) in cirrhotic rat treated with $600 \mathrm{mg} / \mathrm{kg}$ body weight HPE-XA-08 in comparison to cirrhotic rat treated with just water diluent. Reversion of cirrhotic liver to normal liver condition in rats treated with HPE-XA-08 was observed. Results from the present study suggest that HPE-XA-08 treatment assisted in the protection from liver cirrhosis and improved the recovery of cirrhotic liver.
\end{abstract}

\section{Introduction}

Chronic liver disease is the ninth leading cause of mortality in Western and developing countries [1]. The disease resulted from chronic proinflammatory injuries, which could cause progressive fibrosis causing the liver to scar and eventually becomes cirrhotic. There are many factors that could lead to chronic liver injury and among them are viral infections such as hepatitis $\mathrm{C}$ infection, chronic alcoholism, and autoimmune diseases and also due to drug or substance abuses. Five percent of persons with liver cirrhosis could progress to develop liver cancer [2].

To date, there is no specific treatment for liver cirrhosis. Sufferers are treated to reduce the complications due to the damaged liver from exacerbating. The treatments are often expensive especially for those in developing countries where there is high rate of liver cirrhosis in the population. Due to these factors, treatment using ethnobotanical approach has gained popularity as an alternative cost-effective approach $[1,3]$. Among the ethnobotanical approaches, the Chinese herbal medicine has been widely applied. It serves as alternative complementary medicine, probably due to the presence of complete pharmacopeia of the herbs established over more than 5000 years of traditional use. Currently, in many healthcare facilities in China, traditional Chinese medicine is being applied in complement to Western medicine [4]. Typical in most traditional Chinese medicines, the formulation consists of multiple herbs concoction. It has been suggested that the combination of herbs with the various combinations of natural ingredients may produce synergistic 
effects and neutralize potential side effects of an individual herb constituent, hence augmenting the effectiveness of a treatment [5-7].

The present study aims to evaluate the preventive and potential curative effects of a decoction containing 10 Chinese medicinal herbs, HPE-XA-08, in which a number of the herbs have been reported to be useful in supporting liver functions and help to ameliorate liver fibrosis/cancer [8-11].

\section{Materials and Methods}

2.1. Plant Extracts. Aqueous extract of HPE-XA-08 containing mixture of 10 Chinese medicinal herbs, comprising fructus Gardenia jasminoides Ellis, Var. radicans (Thunb.) Makino, Artemisia scoparia Waldst. Et Kit, radix Rheum tanguticum Maxim. Ex Balf, radix Scutellaria baicalensis Georgi, fructus Ligustrum lucidum Ait, cortex Phellodendron amurense Rupr., Poria cocoa (Schw.) Wolf, radix Bupleurum B. scorzoneraefolium Willd, and flos Chrysanthemum morifolium Ramat and herbs Tarxacum mongolicum Hand, Mazz were prepared according to propriety extraction method of Herbitec (M) Sdn. Bhd. The aqueous extract was filtered to remove all the coarse residues, sterile filtered, and kept at $-20^{\circ} \mathrm{C}$ until being needed for the study.

2.2. Animals. Male Sprague-Dawley (SD) rats aged between 7 and 10 weeks were kept in the University Malaya animal breeding house. Animals were fed with standard pellet diet and water ad libitum at $20-25^{\circ} \mathrm{C}$. All animal handlings and protocols were in accordance with the institutional guidelines for laboratory animals (Ethic Reference Number PM/27/08/2011/MAA(R)).

2.3. Evaluation for Prevention and Treatment of TAA-Induced Liver Cirrhosis by HPE-XA-08. Preventive effects for liver cirrhosis conferred by HPE-XA-08 were evaluated using the $\mathrm{SD}$ rats. Rats were assigned to 4 groups, namely, Group 1P: control group 1 (normal saline $+\mathrm{H}_{2} \mathrm{O}$ ), Group 2P: control group 2 (normal saline + HPE-XA-08), Group 3P: TAA control group (TAA $+\mathrm{H}_{2} \mathrm{O}$ ), and Group 4P: treated group (TAA + HPE-XA-08 treatment; pretreatment with HPE-XA$08,300 \mathrm{mg} / \mathrm{kg}$ ). Rats from Groups $1 \mathrm{P}$ and $2 \mathrm{P}$ were injected intraperitoneally (ip) with normal saline while Groups 3P and $4 \mathrm{P}$ were injected with thioacetamide (TAA) at a dose of $200 \mathrm{mg} / \mathrm{kg}$ twice weekly for 12 weeks. HPE-XA-08 was administered orally at $300 \mathrm{mg} / \mathrm{kg}$ via a stomach tube to rats in Groups 2P and 4P daily, up to 12 weeks, while Group $1 \mathrm{P}$ and Group 3P were fed with distilled water. For the evaluation of HPE-XA-08 treatment on TAA-induced liver cirrhosis, 3 rat groups were assigned, namely, Group 1T: normal control group, Group 2T: cirrhosis control group, and Group 3T: treatment group (posttreatment with HPE-XA-08, $600 \mathrm{mg} / \mathrm{kg}$ ). Liver cirrhosis was induced in rats of Groups 2T and $3 \mathrm{~T}$ by inoculating TAA at $200 \mathrm{mg} / \mathrm{kg}$ intraperitoneally, twice weekly, for 12 weeks. Rats of Group 1T that served as normal control were inoculated with normal saline. After 12 weeks, the TAA was stopped and the rats from Group 3T were treated with $600 \mathrm{mg} / \mathrm{kg}$ HPE-XA-08 as described above while rats of Groups $1 \mathrm{~T}$ and $2 \mathrm{~T}$ were treated with distilled water of the equivalent volume. Blood was collected from each rat before and after the treatment. The treatment was performed for 30 days.

2.4. Hepatic Biochemical Evaluation. Blood of each rat was collected via tail vein before the treatment and at the end of the treatment regime. The blood was collected in tube containing EDTA and analyzed for the liver function enzymes serum alanine transaminase (ALT), aspartate transaminase (AST), alkaline phosphatase (ALP), and gamma-glutamyl transferase (GGT) in the Clinical Diagnostic Laboratory (CDL) at University Malaya Medical Centre (UMMC). Immediately after the final blood collection, the rats were euthanized and the organs were harvested for histological investigation.

2.5. Histopathological Analysis. Liver samples harvested from the rats were washed with the normal saline and immediately fixed in $10 \%$ buffered neutral formalin for 48 hours. Samples were then embedded in paraffin wax. Sections of 5-micron thickness were prepared, processed in alcohol-xylene series, and stained with alum-haematoxylin and eosin, prior to histopathological examination.

2.6. Statistical Analysis. Results were presented as mean \pm SEM of six animals in each group. The data were subjected to one-way ANOVA followed by Bonferroni's posttest. $p<$ 0.05 was considered statistically significant. Analysis was performed using GraphPad Prism version 4.00 for Windows (GraphPad Software, USA).

\section{Results}

3.1. Prevention of TAA-Induced Liver Cirrhosis. The effects of HPE-XA- 08 on serum ALT, AST, ALP, and GGT and bilirubin activities in rats from all treatment groups were shown in Table 1. It is observed that the serum ALT and AST activities in all rat groups, Groups $1 \mathrm{P}, 2 \mathrm{P}, 3 \mathrm{P}$, and $4 \mathrm{P}$, did not show any significant differences following the preventive treatment regime. It was noted that the serum ALT activity in rat group treated with HPE-XA-08 showed lower ALT activity with value of $76.0 \pm 4.416 \mathrm{IU} / \mathrm{L}$ (Group $2 \mathrm{P}$ ) and $69.40 \pm 7.756 \mathrm{IU} / \mathrm{L}$ (Group 4P) in comparison to $80.0 \pm 4.175$ IU/L (Group 1P) and $88.5 \pm 12.75 \mathrm{IU} / \mathrm{L}$ (Group 3P). For serum AST level, the TAA control group (Group 3P) showed the lowest level with value of $215.0 \pm 13.64 \mathrm{IU} / \mathrm{L}$, followed by Groups 4P, 2P, and $1 \mathrm{P}$ with values of $222.4 \pm 5.715 \mathrm{IU} / \mathrm{L}, 223.3 \pm 11.16 \mathrm{IU} / \mathrm{L}$, and $237.3 \pm 12.84 \mathrm{IU} / \mathrm{L}$, respectively. Serum ALP level in rat groups injected with TAA (Groups 3P and 4P) were higher in comparison to those injected with normal saline only (Groups 1P and 2P). The highest level of ALP was observed in Group 3P with value of $375.0 \pm 10.41 \mathrm{IU} / \mathrm{L}$. The ALP level in rat group injected with TAA and treated with HPE-XA08 (Group 4P) was observed to be lower than those treated with water (Group 3P) with value of $277 \pm 4.722 \mathrm{IU} / \mathrm{L}$. The serum GGT levels in rat groups injected with TAA (Groups $3 \mathrm{P}$ and $4 \mathrm{P}$ ) were significantly higher in comparison to the control groups (Groups $1 \mathrm{P}$ and $2 \mathrm{P}$ ). Serum GGT levels in TAA control group (Group 3P) were the highest with value 
TABLE 1: Effect of HPE-XA-08 on blood biochemicals related to liver damage (ALT, AST, ALP, and GGT) for observation of protective activity.

\begin{tabular}{|c|c|c|c|c|}
\hline Group & ALT (IU/L) & AST (IU/L) & $\operatorname{ALP}(\mathrm{IU} / \mathrm{L})$ & GGT (IU/L) \\
\hline Normal saline $+\mathrm{H}_{2} \mathrm{O}(1 \mathrm{P})$ & $80.00 \pm 4.175$ & $237.3 \pm 12.84$ & $173.6 \pm 7.807$ & $2.286 \pm 0.4206$ \\
\hline Normal saline + HPE-XA-08 $(2 \mathrm{P})^{\#}$ & $76.00 \pm 4.416$ & $223.3 \pm 11.16$ & $170.3 \pm 23.68$ & $3.750 \pm 0.4787$ \\
\hline $\mathrm{TAA}+\mathrm{H}_{2} \mathrm{O}(3 \mathrm{P})$ & $88.50 \pm 12.75$ & $215.0 \pm 13.64$ & $375.0 \pm 10.41^{*}$ & $37.50 \pm 1.857^{*}$ \\
\hline $\mathrm{TAA}+\mathrm{HPE}-\mathrm{XA}-08(4 \mathrm{P})^{+}$ & $69.40 \pm 7.756$ & $222.4 \pm 5.715$ & $277.0 \pm 4.722$ & $30.80 \pm 3.980$ \\
\hline
\end{tabular}

${ }^{*}$ Levels of enzymes between $1 \mathrm{P}$ and $3 \mathrm{P}$ were compared to show the effects of TAA on the level of liver enzymes; $*$ indicates $p<0.05$.

${ }^{\#}$ Levels of enzymes between $1 \mathrm{P}$ and $2 \mathrm{P}$ were compared to show the effects of HPE-XA-08 on the level of liver enzymes but did not show statistically significant value $(p>0.05)$.

${ }^{+}$Levels of enzymes between $3 \mathrm{P}$ and $4 \mathrm{P}$ were compared to show the effects of HPE-XA-08 on preventing TAA-induced liver cirrhosis but did not show statistically significant value $(p>0.05)$.

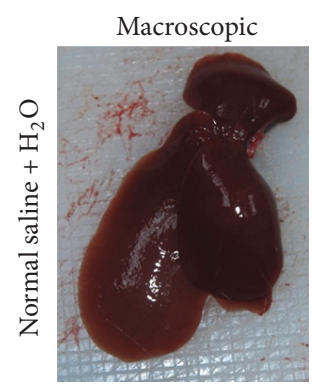

(a)

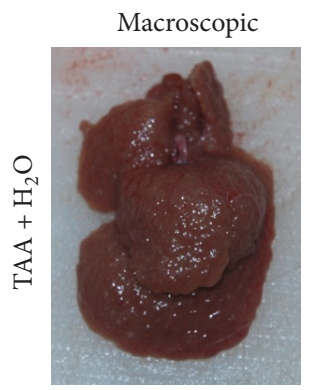

(e)

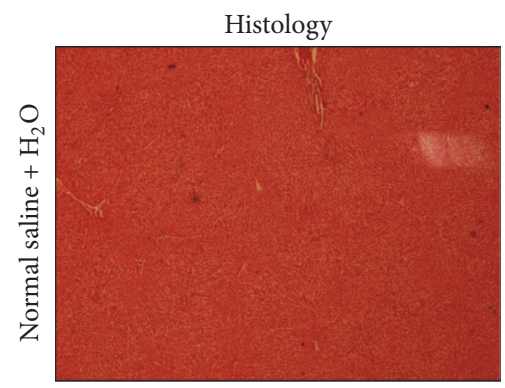

(b)

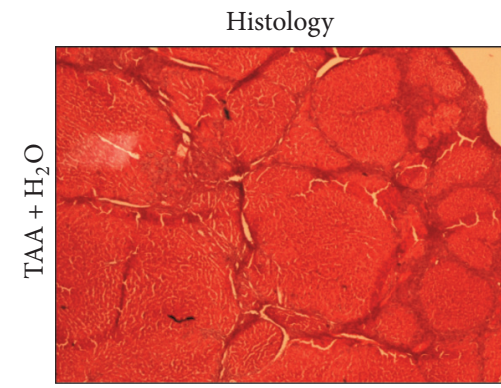

(f)

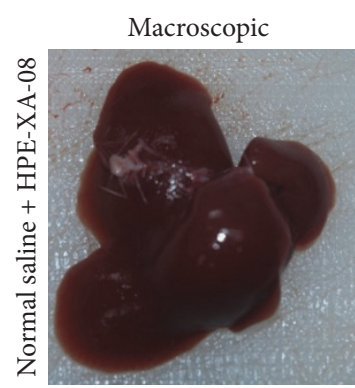

(c)

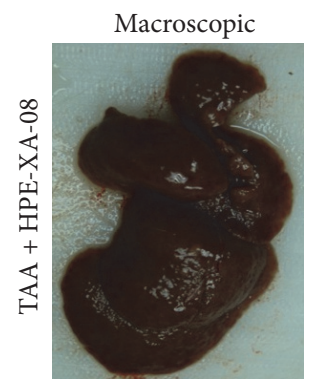

(g)

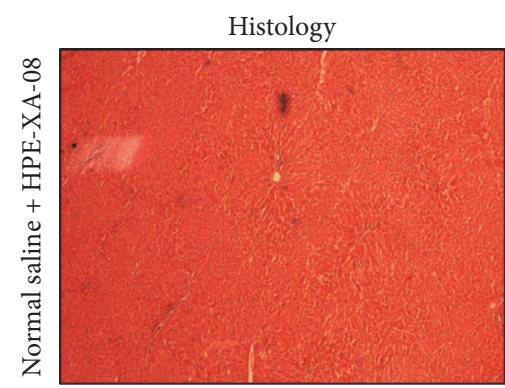

(d)

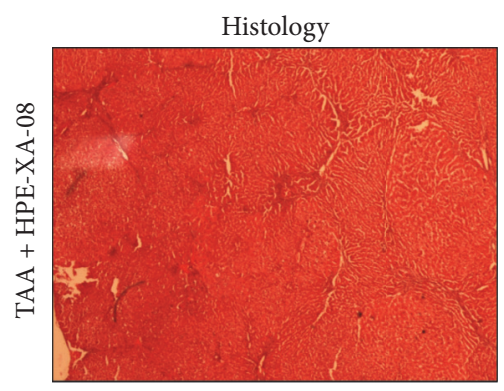

(h)

FIGURE 1: Macroscopic and histologic analysis of liver harvested from rats. Liver samples were harvested from rats inoculated with normal saline and treated with water (a), inoculated with normal saline and treated with HPE-XA-08 (c), inoculated with TAA (200 mg/kg) twice weekly and treated with water (e), and inoculated with TAA and treated with HPE-XA-08 (g). The sections from harvested liver were stained with hematoxylin-eosin, respectively $(b, \mathrm{~d}, \mathrm{f}, \mathrm{h})$.

of $37.50 \pm 1.857 \mathrm{IU} / \mathrm{L} \mathrm{mmol} / \mathrm{L}$. The rat group $4 \mathrm{P}(\mathrm{TAA}+\mathrm{HPE}-$ XA-08 treatment) showed lower GGT level when compared to the TAA control group (Group 3P) with value of $30.80 \pm$ 3.980 IU/L mmol/L.

Histological examination of liver section of rats from the control groups (Groups $1 \mathrm{P}$ and $2 \mathrm{P}$ ) showed that the liver was dark red in color, with smooth homogenous surface texture (Figures 1(a) and 1(c)). The liver of rats from Group 3P was observed to be slightly brownish in color. The surface was rough, irregular with highly nodulated morphology (Figure $1(\mathrm{e})$ ). In the rat group of TAA + HPE-XA-08 treatment (Group 4P), although the color of the liver was noted as dark brown, the surface of the liver was smoother and the nodules were not as distinct as the liver of rat group 3P (Figure $1(\mathrm{~g})$ ). The liver section of rats from control groups (Groups 1P and $2 \mathrm{P}$ ) showed a homogenous distribution of hepatic cells devoid of extracellular matrix protein (ECM; Figures 1(b) and $1(\mathrm{~d})$ ). The liver section of TAA-treated control group (Group 3P) exhibited marked presence of ECM extending through the hepatic lobules and displayed as large fibrous septa (Figure 1(f)). However, no special histological staining was performed to determine the component of the septa. In the liver section of the TAA + HPE-XA-08 treatment group (Group 4P), the presence of ECM was almost not detected. The fibrous septa were not present as those observed in Figure 1(f), although trace of injury can still be observed (Figure 1(h)).

3.2. Treatment of TAA-Induced Liver Cirrhosis. The possibility for HPE-XA-08 to reverse liver cirrhosis in rats was also evaluated. All rats were inoculated with TAA to induce liver cirrhosis, except for rat group 1T which was inoculated with normal saline to serve as control group (normal rat). Following 12 weeks of induction of liver cirrhosis with TAA, 


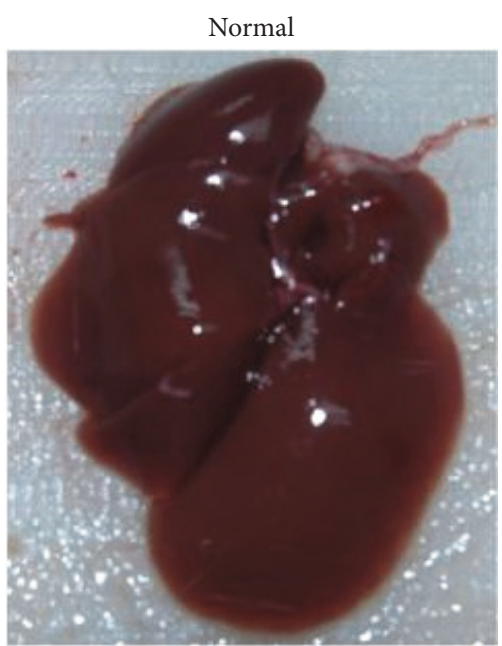

(a)

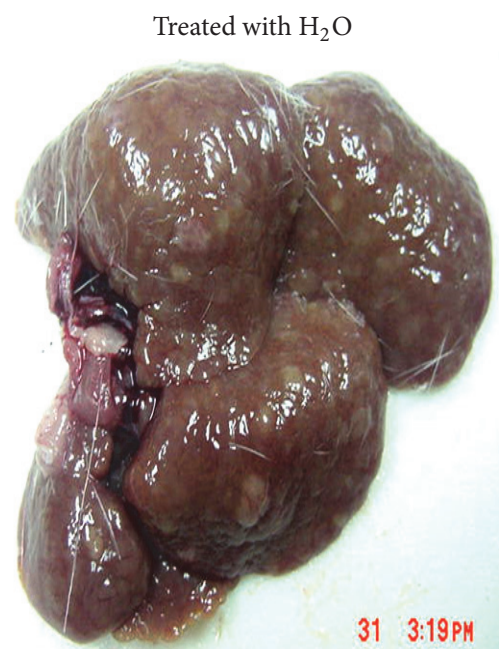

(b)

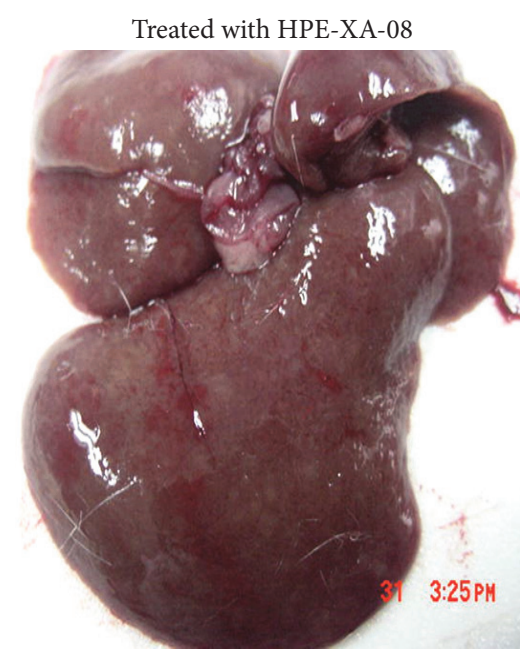

(c)

FIGURE 2: Macroscopic analysis of cirrhotic liver following treatment regime. Liver cirrhosis was induced by TAA for 12 weeks. After 12 weeks, the TAA was discontinued and rats were treated with either water (b) or HPE-XA-08 (c). Normal rat was included as negative control and treated with water (a).

the rats were either treated with distilled water (Group $2 \mathrm{~T}$ ) or with $600 \mathrm{mg} / \mathrm{kg}$ HPE-XA-08 for 30 days (Group 3T). The hepatic biochemical activities in normal and cirrhotic rats were evaluated before and after the treatment regime. The hepatic enzyme levels of ALT, AST, ALP, and GGT were measured (Table 2). It was noted that the AST, ALP, and GGT levels decreased after the 30-day treatment in all rat groups. The ALT level, however, was noted to increase in the cirrhotic rat groups (Groups $2 \mathrm{~T}$ and $3 \mathrm{~T}$ ) following the 30 -day treatment with increment from $62 \pm 0.636 \mathrm{IU} / \mathrm{L}$ to $75 \pm 0.577 \mathrm{IU} / \mathrm{L}$ in Group $2 \mathrm{~T}$ and $63 \pm 1.202 \mathrm{IU} / \mathrm{L}$ to 69 $\pm 0.441 \mathrm{IU} / \mathrm{L}$ in Group 3T. Withdrawal of TAA helped to significantly reduce the level of ALP and GGT in Group $2 \mathrm{~T}$ although the rats were treated with just water. In rats treated with HPE-XA-08 (Group 3T), further decrease of ALP and GGT levels with reduction from $368 \pm 0.882 \mathrm{IU} / \mathrm{L}$ to $102 \pm$ $2.333 \mathrm{IU} / \mathrm{L}$ and $39 \pm 0.296 \mathrm{IU} / \mathrm{L}$ to $15 \pm 0.441 \mathrm{IU} / \mathrm{L}$ for ALP and GGT, respectively, was observed. The AST level was reduced significantly following treatment with HPE-XA-08 with reduction from $179 \pm 0.882 \mathrm{IU} / \mathrm{L}$ to $158 \pm 0.882 \mathrm{IU} / \mathrm{L}$. Unlike those treated with HPE-XA-08, the AST level in the cirrhotic rat treated with water was $180 \pm 1.155 \mathrm{IU} / \mathrm{L}$ and $175 \pm 0.667 \mathrm{IU} / \mathrm{L}$ before and after treatment, respectively. Macroscopic observation of the liver from the normal control rat (Group 1T) showed the liver to be dark red in color with smooth homogenous surface (Figure 2(a)). In the cirrhotic control group treated with water (Group 2T), although the TAA has been discontinued, the liver was slightly brownish with macronodular structure and irregular nonhomogenous surface (Figure 2(b)). For the cirrhotic rats treated with HPE$\mathrm{XA}-08$, the color of the liver was more reddish compared to the group treated with only water (Figure 2(c)). The liver surface showed no obvious nodular structure and the surface of the liver was comparable to the normal liver.

\section{Discussion}

Hepatic cirrhosis, a consequence of hepatic fibrosis, is characterized by exaggerated production of extracellular matrix properties (ECM) [12]. Hepatic cirrhosis induced by thioacetamide (TAA) lead to steatosis, which is associated with the aggravation of lipid peroxidation and depletion of antioxidant status [13].

In the present study, liver damage is manifested by increases in serum ALP, GGT, and bilirubin levels. TAAinduced liver cirrhosis, however, was usually not characterized by high serum ALT and AST, unlike those intoxicated by $\mathrm{CCl}_{4}$ and paracetamol $[14,15]$. Further, it has been reported that the severity of liver injury does not correlate with the level of liver enzyme elevation [16]. From our observation on the preventive effects of HPE-XA-08 on liver cirrhosis, although the differences between the level of liver enzymes in those untreated and those treated with HPE-XA-08 were not significant, except for level of GGT, the macroscopic and histologic examinations supported that treatment with HPEXA-08 protected the liver against severe damage. Based on our findings, ALP and GGT could be employed as indicator in evaluating liver damage induced by TAA; however, the enzyme levels may not corroborate with the physical condition of the liver. The histological observation of the liver of rats given TAA was consistent with that reported by Bruck et al. [12], where increase in ECM was noted. In this study, however, we did not determine the mechanisms of action of the herbal concoction (HPE-XA-08) in providing the protective effects on the liver against induced damage. We postulated that the HPE-XA-08 decoction may affect the collagen content in the liver by either increasing the collagen synthesis, or reducing the collagen degradation, which was evidenced by the resolution of the fibrotic lesion and ECM in 


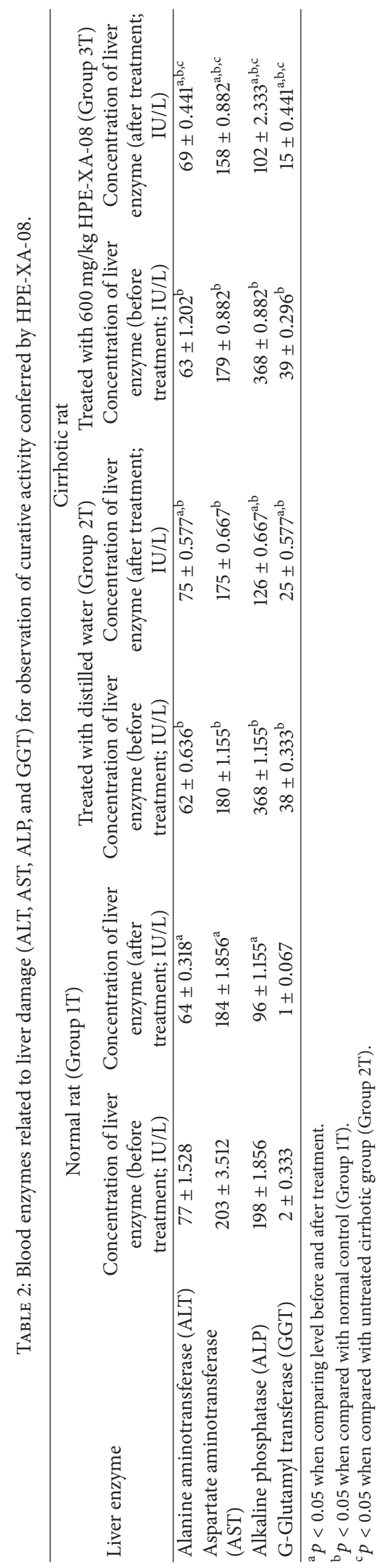


the liver of HPE-XA-08-treated rat observed macroscopically and histologically. The liver harvested from cirrhotic rat treated with HPE-XA-08 showed accelerated resolution of existing fibrosis. The morphology of the liver has reverted to almost comparable to the normal liver (Figure 2(c)). This finding was consistent with reports by Chen et al. [17] and Park et al. [18] that demonstrated that 2 out of 10 components within HPE-XA-08 and Gardenia jasminoides and Scutellaria baicalensis play a role in attenuation of collagen accumulation and apoptosis of hepatic stellate cells (HSCs), respectively, which help in resolution of liver fibrosis. Other than that, the resolution of liver damage may also be assisted by extract of Ligustrum lucidum which was reported to induce apoptosis and cell senescence in human hepatocellular carcinoma [19].

Liver fibrosis and cirrhosis were earlier thought of as irreversible processes. However, recent clinical and experimental evidences suggest that the process can be reversed $[2,20]$. To assess the potential curative effects of HPE-XA-08 on liver cirrhosis, the hepatotoxic TAA was removed during the treatment process. We noted that although TAA has been withdrawn for 30 days, the liver nodulation could still be observed (Figure 2(b)); however, the liver condition was not as nodulated as observed in the liver with the presence of TAA (Figure 1(e)) suggesting possible arrest of progressive liver damage. Previous report showed that TAA-induced steatosis was due to accumulation of lipids within the hepatocytes [21]. Our observation in the rats' cirrhotic liver was consistent with this report (Figure 2(b)), where lipid nodules could be observed in the cirrhotic liver of rats treated with water $\left(\mathrm{H}_{2} \mathrm{O}\right)$. Amelioration of hyperlipidemic liver observed in our study could be contributed by constituents present in Chrysanthemum morifolium and Artemisia scoparia (within HPE-XA-08 decoction), which has been described to attenuate high-fat milk-induced fatty liver and reduce the liver lipid accumulation, respectively $[22,23]$. In the assessment of liver enzyme, the level obtained before the treatment was used as a baseline to be compared to the enzyme level after the treatment. It was noted that the levels of liver enzyme in normal rat decreased after 30 days of treatment with water. This could be related to the age of the rat, since age does affect the liver enzymes [24], which indicated that observation of liver enzyme level alone was insufficient in evaluating hepatoprotective effects conferred by any herbal supplement and needed to be complemented with biopsy or histology.

Most of the previous studies on the antihepatofibrotic were performed on single plant/herb extract. In this study, the hepatocellular injury and fibrosis in rats were treated orally with decoction consisting of 10 medicinal Chinese herbs, named HPE-XA-08. Other than the 5 plants/herbs within HPE-XA-08 that have been mentioned above, the remaining 4 plants/herbs in the decoction, Phellodendron amurense, Poria cocoa, Bupleurum B. scorzoneraefolium, and Tarxacum mongolicum, scientifically were reported to exert anticarcinogenic and antitumor properties [25-28]. Based on our findings, we conclude that the decoction consisting of the 10 medicinal Chinese herbs, named HPE-XA-08, possesses potential hepatoprotective and curative effects against damaged liver. Further investigations, however, are needed to identify the hepatoprotective mechanisms conferred by this aqueous herbal preparation. The present finding provides scientific evidence that the mixture of these 10 Chinese traditional herbs preparation possessed ethnomedicinal properties in maintaining liver function and could be developed further as ethnomedicine for liver injuries.

\section{List of Abbreviations}

ALT: Alanine aminotransferase

ALP: Alkaline phosphatase

AST: Aspartate aminotransferase

ECM: Extracellular matrix

GGT: Gamma-glutamyl transferase

$\mathrm{H}_{2} \mathrm{O}$ : Water

IP: Intraperitoneal

SEM: Standard error of mean

TAA: Thioacetamide.

\section{Competing Interests}

Tong-Hye Lim is the herbalist in Herbitec (M) Sdn Bhd. Sazaly AbuBakar is the Scientific Consultant for Herbitec (M) Sdn Bhd. Nor Aziyah Mat-Rahim and Nur-Asyura NorAmdan have no conflict of interests.

\section{Acknowledgments}

This study received grant support from Herbitec (M) Sdn Bhd. The authors also thank Herbitec (M) Sdn Bhd for providing continuous supply of HPE-XA-08 extract and Professor Dr. Mahmood Ameen Abdulla, Department of Molecular Medicine, Faculty of Medicine, University Malaya (UM), for his guidance and assistance with the animal study and histology.

\section{References}

[1] T. S. Mohamed Saleem, C. M. Chetty, S. Ramkanth, V. S. T. Rajan, K. M. Kuma, and K. Gauthman, "Hepatoprotective herbs-a review," International Journal of Research in Pharmaceutical Sciences, vol. 1, pp. 1-5, 2010.

[2] A. M. Elsharkawy, F. Oakley, and D. A. Mann, "The role and regulation of hepatic stellate cell apoptosis in reversal of liver fibrosis," Apoptosis, vol. 10, no. 5, pp. 927-939, 2005.

[3] S. P. Thyagarajan, S. Jayaram, V. Gopalakrishnan, R. Hari, P. Jeyakumar, and M. S. Sripathi, "Herbal medicines for liver diseases in India," Journal of Gastroenterology and Hepatology, vol. 17, supplement 3, pp. S370-S376, 2002.

[4] “Traditional Chinese medicine: in depth," December 2013, https://nccih.nih.gov/health/whatiscam/chinesemed.htm.

[5] D. Bensky and A. Gamble, Chinese Herbal Medicine in Materia, Eastland Press, Seattle, Wash, USA, 1993.

[6] H. Sharma, "Phytochemical synergism: beyond the active ingredient model," Journal of Alternative Therapies in Clinical Practice, vol. 4, pp. 91-96, 1997.

[7] Y. P. Zhu, Chinese Material Medica: Chemistry, Pharmacology and Applications, Harwood Academic Publishers, Amsterdam, The Netherlands, 1998. 
[8] Y. Zhao, H. Li, Z. Gao, Y. Gong, and H. Xu, "Effects of flavonoids extracted from Scutellaria baicalensis Georgi on hemin-nitrite$\mathrm{H} 2 \mathrm{O} 2$ induced liver injury," European Journal of Pharmacology, vol. 536, no. 1-2, pp. 192-199, 2006.

[9] T.-Y. Lee, H.-H. Chang, J.-H. Chen, M.-L. Hsueh, and J.-J. Kuo, "Herb medicine Yin-Chen-Hao-Tang ameliorates hepatic fibrosis in bile duct ligation rats," Journal of Ethnopharmacology, vol. 109, no. 2, pp. 318-324, 2007.

[10] E. Yarnell and K. Abascal, "Dandelion (Taraxacum officinale and T. mongolicum)," Journal of Intergrative Medicine, vol. 8, no. 2, pp. 35-38, 2009.

[11] X.-M. Wang, X.-Q. Hou, Y.-Q. Zhang, and Y. Li, "Distribution pattern of genuine species of rhubarb as traditional Chinese medicine," Journal of Medicinal Plants Research, vol. 4, no. 18, pp. 1865-1876, 2010.

[12] R. Bruck, O. Genina, H. Aeed et al., "Halofuginone to prevent and treat thioacetamide-induced liver fibrosis in rats," Hepatology, vol. 33, no. 2, pp. 379-386, 2001.

[13] A. Cruz, F. J. Padillo, E. Torres et al., "Melatonin prevents experimental liver cirrhosis induced by thioacetamide in rats," Journal of Pineal Research, vol. 39, no. 2, pp. 143-150, 2005.

[14] A. K. Gupta and N. Misra, "Hepatoprotective activity of aqueous ethanolic extract of chamomile capitula in paracetamol intoxicated albino rats," American Journal of Pharmacology and Toxicology, vol. 1, no. 1, pp. 17-20, 2006.

[15] Y. Feng, K.-Y. Siu, X. Ye et al., "Hepatoprotective effects of berberine on carbon tetrachloride-induced acute hepatotoxicity in rats," Chinese Medicine, vol. 5, article 33, 2010.

[16] A. O. Shakil, C. Conry-Cantilena, H. J. Alter et al., "Volunteer blood donors with antibody to hepatitis $\mathrm{C}$ virus: clinical, biochemical, virologic, and histologic features," Annals of Internal Medicine, vol. 123, no. 5, pp. 330-337, 1995.

[17] Y.-H. Chen, T. Lan, J. Li et al., "Hepatoprotective activity of aqueous ethanolic extract of chamomile capitula in paracetamol intoxicated albino rats," World Journal of Gastroenterology, vol. 18, no. 48, pp. 7158-7165, 2012.

[18] E.-J. Park, Y.-Z. Zhao, L. Lian, Y.-C. Kim, and D. H. Sohn, "Skullcapflavone I from Scutellaria baicalensis induces apoptosis in activated rat hepatic stellate cells," Planta Medica, vol. 71, no. 9, pp. 885-887, 2005.

[19] B. Hu, Q. Du, S. Deng et al., "Ligustrum lucidum Ait. fruit extract induces apoptosis and cell senescence in human hepatocellular carcinoma cells through upregulation of p21," Oncology Reports, vol. 32, no. 3, pp. 1037-1042, 2014.

[20] A. Pellicoro, P. Ramachandran, and J. P. Iredale, "Reversibility of liver fibrosis," Fibrogenesis and Tissue Repair, vol. 5, supplement, p. S26, 2012.

[21] A. A. Amali, R. D. Rekha, C. J.-F. Lin et al., “Thioacetamide induced liver damage in zebrafish embryo as a disease model for steatohepatitis," Journal of Biomedical Science, vol. 13, no. 2, pp. 225-232, 2006.

[22] Y. Cui, X. Wang, J. Xue, J. Liu, and M. Xie, "Chrysanthemum morifolium extract attenuates high-fat milk-induced fatty liver through peroxisome proliferator-activated receptor $\alpha$-mediated mechanism in mice," Nutrition Research, vol. 34, no. 3, pp. 268$275,2014$.

[23] Z. Q. Wang, X. H. Zhang, Y. Yu et al., "Artemisia scoparia extract attenuates non-alcoholic fatty liver disease in diet-induced obesity mice by enhancing hepatic insulin and AMPK signaling independently of FGF21 pathway," Metabolism: Clinical and Experimental, vol. 62, no. 9, pp. 1239-1249, 2013.
[24] P. D. Wilson, R. Watson, and D. L. Knook, "Effects of age on rat liver enzymes. A study using isolated hepatocytes, endothelial and Kupffer cells," Gerontology, vol. 28, no. 1, pp. 32-43, 1982.

[25] S. C. Sigstedt, C. J. Hooten, M. C. Callewaert et al., "Evaluation of aqueous extracts of Taraxacum officinale on growth and invasion of breast and prostate cancer cells," International Journal of Oncology, vol. 32, no. 5, pp. 1085-1090, 2008.

[26] M. A. James, H. Fu, Y. Liu, D.-R. Chen, and M. You, "Dietary administration of berberine or Phellodendron amurense extract inhibits cell cycle progression and lung tumorigenesis," Molecular Carcinogenesis, vol. 50, no. 1, pp. 1-7, 2011.

[27] A. Li, "Chinese medicine composition for treating cancer," U.S. Patent US 8414937, 2013.

[28] B. Y.-K. Law, J.-F. Mo, and V. K.-W. Wong, "Autophagic effects of Chaihu (dried roots of Bupleurum Chinense DC or Bupleurum scorzoneraefolium WILD)," Chinese Medicine, vol. 9, article 21, 2014. 


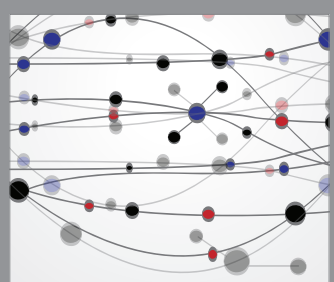

The Scientific World Journal
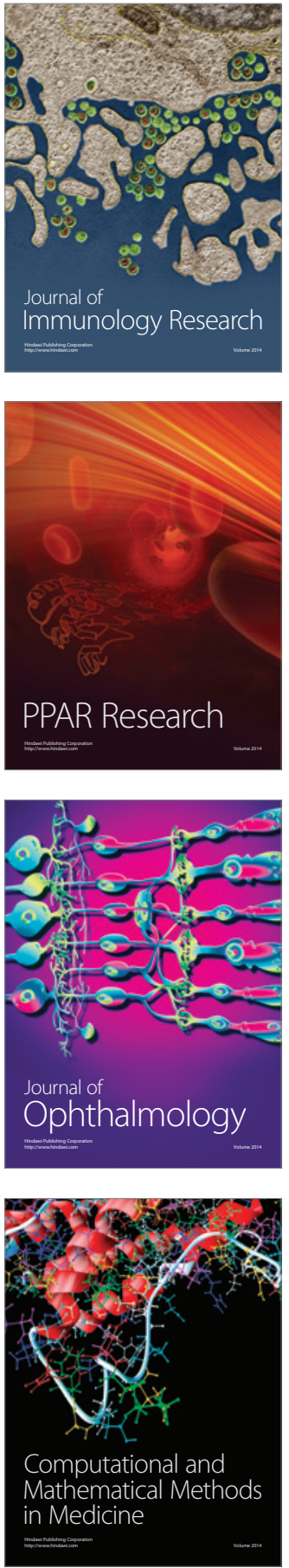

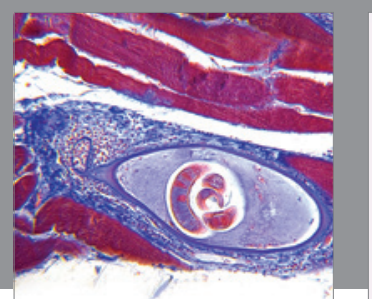

Gastroenterology Research and Practice
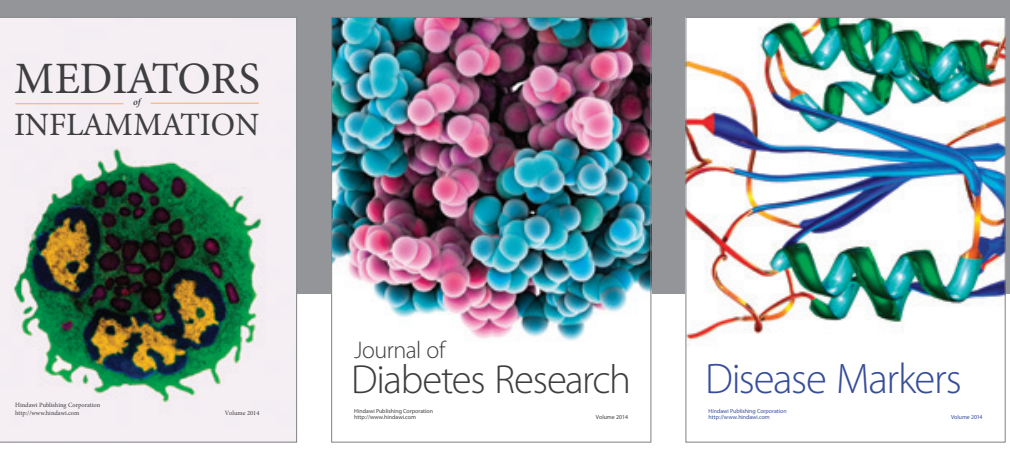

Disease Markers

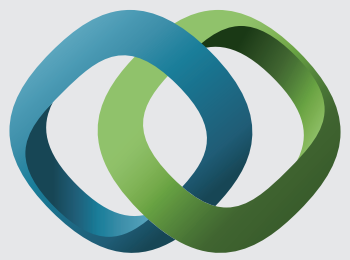

\section{Hindawi}

Submit your manuscripts at

https://www.hindawi.com
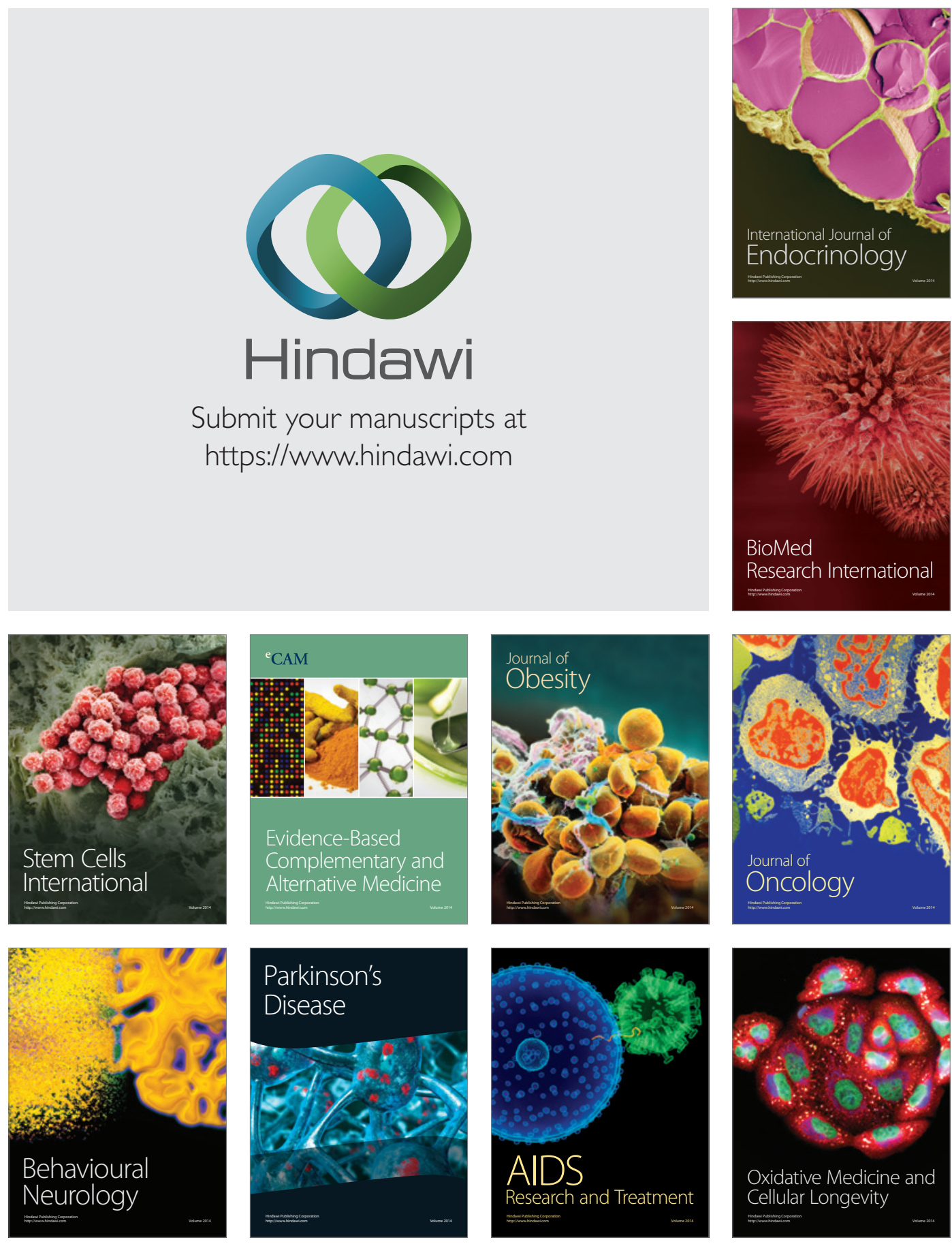